\title{
GRAVITATIONAL BILLIARDS, DUALITIES AND HIDDEN SYMMETRIES
}

\author{
H. Nicolai \\ Max-Planck-Institut für Gravitationsphysik, Albert-Einstein-Institut, \\ Am Mühlenberg 1, D-14476 Golm, Germany
}

\begin{abstract}
The purpose of this article is to highlight the fascinating, but only very incompletely understood relation between Einstein's theory and its generalizations on the one hand, and the theory of indefinite, and in particular hyperbolic, Kac Moody algebras on the other. The elucidation of this link could lead to yet another revolution in our understanding of Einstein's theory and attempts to quantize it.
\end{abstract}

\section{Introduction}

As we look back 90 years to take stock of what has been achieved since Einstein explained gravity in terms of spacetime geometry and its curvature, the progress is impressive. Even more impressive is the wealth of structure contained in Einstein's equations which has been revealed by these developments. Major progress has been made concerning

- Exact solutions (Schwarzschild, Reissner-Nordström, Kerr, axisymmetric stationary solutions,...)

- Cosmological applications (standard FRW model of cosmology, inflationary universe,...)

- Mathematical developments (singularity theorems, black hole uniqueness theorems, studies of the initial value problem, global stability results,...)

- Conceptual developments (global structure and properties of spacetimes, horizons, black hole entropy, quantum theory in the context of cosmology, holography,...) 
- Canonical formulations (Dirac's theory of constrained systems, ADM formalism, Ashtekar's variables,...)

- Higher dimensions (Kaluza Klein theories, brane worlds,...)

- Unified theories 'beyond' Einstein (supergravity, superstrings, supermembranes and $\mathrm{M}($ atrix) theory,...)

- Quantizing gravity (perturbative and canonical quantization, path integral approaches, dynamical triangulations, spin networks and spin foams,...)

All these subjects continue to flourish and are full of promise for further and exciting developments (hinted at by the dots in the above list). No doubt many of them will be discussed and elaborated in other contributions to this volume. In this article, we will concentrate on yet another line of research that evolved out of the study of Einstein's equations and its locally supersymmetric extensions, and which points to another deep, and still mostly unexplored property of Einstein's theory. It may well be that the main discoveries in this direction still remain to be made, and that, once they have been made, they will also have a profound impact on attempts to quantize Einstein's theory (or some bigger theory containing it). This is the subject of

\section{- Hidden symmetries and dualities}

The first hint of these symmetries appeared in Ref. 1 , where a transformation between two static solutions of Einstein's equations was given, which in modern parlance is nothing but a $T$-duality transformation. A decisive step was Ehlers' discovery in 1957 of a solution generating symmetry, ${ }^{2}$ nowadays known as the 'Ehlers $S L(2, \mathbb{R})$ symmetry' which acts on certain classes of solutions admitting one Killing vector. In 1970, R. Geroch demonstrated the existence of an infinite dimensional extension of the Ehlers group acting on solutions of Einstein's equations with two commuting Killing vectors (axisymmetric stationary solutions). ${ }^{3}$ In the years that followed, the Geroch group was extensively studied by general relativists with the aim of developing 'solution generating techniques' (see ${ }^{4,5}$ and references therein for an entrée into the literature). The field received new impetus with the discovery of 'hidden symmetries' in supergravities, most notably the exceptional $E_{7(7)}$ symmetry of maximal $N=8$ supergravity. ${ }^{6}$ These results showed that the Ehlers and Geroch groups were but special examples of a more general phenomenon. ${ }^{7,8,9,10,11}$ With the shift of emphasis from solution generating techniques to the Lie algebra and the 
group theoretical structures underlying these symmetries, it became clear that the Geroch symmetry is a non-linear and non-local realization of an affine Lie group (a loop group with a central extension), with Lie algebra $A_{1}^{(1)}=\widehat{\mathfrak{s}(2, \mathbb{R})_{c e}}$. This completed earlier results by general relativists who had previously realized 'half' of this affine symmetry in terms of 'dual potentials'. ${ }^{12}$ Likewise, generalizations of Einstein's theory, and in particular its locally supersymmetric extensions were shown to possess similar infinite dimensional symmetries upon reduction to two dimensions. These results also provided a direct link to the integrability of these theories in the reduction to two dimensions, i.e. the existence of Lax pairs for the corresponding equations of motion. ${ }^{13,14,9}$

All these duality invariances of Einstein's theory and its extensions apply only to certain truncations, but do not correspond to properties of the full theory, or some extension thereof. Our main point here will be to review and highlight the evidence for even larger symmetries which would not require any truncations, and whose associated Lie algebras belong to a special class of infinite dimensional Lie algebras, namely the so-called indefinite Kac Moody Algebras. ${ }^{15,16,17}$ We will discuss two examples of such Lie algebras, namely the rank three algebra $A E_{3},{ }^{18}$ which is related to Einstein's theory in four dimensions, and secondly (but only very briefly), the maximal rank 10 algebra $E_{10}$, which is singled out from several points of view, and which is related to maximal $D=11$ supergravity. ${ }^{19}$ We can thus phrase the central open question as follows:

Is it possible to extend the known duality symmetries of Einstein's equations to the full non-linear theory without any symmetry reductions?

A perhaps more provocative, way to pose the question is

\section{Is Einstein's theory integrable?}

In this form, the question may indeed sound preposterous to anyone with even only a passing familiarity with the complexities of Einstein's equations, which are not only the most beautiful, but also the most complicated partial differential equations in all of known mathematical physics. What is meant here, however, is not the usual notion of integrability in the sense that one should be able to write down the most general solution in closed form. Rather, it is the 'mappability' of the initial value problem for Einstein's theory, or some $\mathrm{M}$ theoretic extension thereof, onto a group theoretical structure that itself is equally intricate, and so infinite in extent that we may never be able to work it out completely, although we know that it 
exists. Even a partial answer to the above question would constitute a great advance, and possibly clarify other unsolved problems of general relativity. To name but one: the 'conserved charges' associated with these Lie algebras would almost certainly be linked to the so far elusive 'observables' of pure gravity (which might better be called 'perennials ${ }^{20}$ ) - which we believe should exist, though no one has ever been able to write down a single one explicitly.

Last but not least, duality symmetries have come to play a prominent role in modern string theory in the guise of $T, S$ and $U$ dualities, where they may provide a bridge to the non-perturbative sector of the theory $\left(\mathrm{see}^{21,22,23}\right.$ and references therein). Here, we will not dwell too much on this side of the story, however, because the duality groups considered in string theory so far are descendants of the finite dimensional Lie groups occurring in $D \geq 4$ supergravity, whereas here we will be mostly concerned with the infinite dimensional symmetries that emerge upon reduction to $D \leq 2$ dimensions, and whose role and significance in the context of string theory are not understood. Still, it seems clear that infinite dimensional symmetries may play a key role in answering the question, what M Theory - the conjectural and elusive non-perturbative and background independent formulation of superstring theory - really is, because that question may well be closely related (or even equivalent) to another one, namely

\section{What is the symmetry underlying $M$ Theory?}

There has been much discussion lately about the maximally extended hyperbolic Kac Moody algebra $E_{10}$ as a candidate symmetry underlying $\mathrm{M}$ Theory, i.e. $D=11$ supergravity and the other maximally supersymmetric theories related to IIA and IIB superstring theory, see $24,25,26,27,28,29$, and ${ }^{30,31}$. A conceptually different proposal was made in ${ }^{32}$, and further elaborated in ${ }^{33,34,35}$, according to which it is the 'very extended' indefinite $\mathrm{KM}$ algebra $E_{11}$ that should be viewed as the fundamental symmetry ( $E_{11}$ contains $E_{10}$, but is no longer hyperbolic, but see ${ }^{36}$ for a discussion of such 'very extended algebras'). A 'hybrid' approach for uncovering the symmetries of $M$-theory combining ${ }^{25}$ and ${ }^{32}$ has been adopted in ${ }^{37,38}$. Although our focus here is mostly on pure gravity in four space-time dimensions and its associated algebra $A E_{3}$, we will very briefly mention these developments in the last section.

Whatever the outcome of these ideas and developments will be, the very existence of a previously unsuspected link between two of the most beautiful concepts and theories of modern physics and mathematics, respectively - 
Einstein's theory of gravity on the one hand, and the theory of indefinite and hyperbolic Kac Moody algebras on the other - is most remarkable and surely has some deep significance.

\section{Known duality symmetries}

We first review the two types of duality symmetries of Einstein's theory that have been known for a long time. The first concerns the linearized version of Einstein's equations and works in any space-time dimension. The second is an example of a non-linear duality, which works only for the special class of solutions admitting two commuting Killing vectors (axisymmetric stationary and colliding plane wave solutions). This second duality is more subtle, not only in that it is non-linear, but in that it is linked to the appearance of an infinite dimensional symmetry.

\subsection{Linearized duality}

The duality invariance of the linearized Einstein equations generalizes the well known duality invariance of electromagnetism in four spacetime dimensions. Recall that Maxwell's equations in vacuo

$$
\partial^{\mu} F_{\mu \nu}=0 \quad, \quad \partial_{[\mu} F_{\nu \rho]}=0
$$

are invariant under $U(1)$ rotations of the complex field strength

$$
\mathcal{F}_{\mu \nu}:=F_{\mu \nu}+i \tilde{F}_{\mu \nu}
$$

with the dual ('magnetic') field strength

$$
\tilde{F}_{\mu \nu}:=\frac{1}{2} \epsilon_{\mu \nu \rho \sigma} F^{\rho \sigma}
$$

The action of this symmetry can be extended to the combined electromagnetic charge $q=e+i g$, where $e$ is the electric, and $g$ is the magnetic charge. The partner of the one-form electric potential $A_{\mu}$ is a dual magnetic one-form potential $\tilde{A}_{\mu}$, obeying

$$
\tilde{F}_{\mu \nu}:=\partial_{\mu} \tilde{A}_{\nu}-\partial_{\nu} \tilde{A}_{\mu}
$$

Observe that this dual potential can only be defined on-shell, when $F_{\mu \nu}$ obeys its equation of motion, which is equivalent to the Bianchi identity for $\tilde{F}_{\mu \nu}$. Consequently, the $U(1)$ duality transformations constitute an onshell symmetry because they are valid only at the level of the equations of motion. The two potentials $A_{\mu}$ and $\tilde{A}_{\mu}$ are obviously non-local functions of one another. Under their exchange, the equations of motion and the 
Bianchi identities are interchanged. Moreover, the equations of motion and the Bianchi identities can be combined into a single equation

$$
\partial^{\mu} \mathcal{F}_{\mu \nu}=0
$$

Analogous duality transformations to the electromagnetic ones exhibited above exist for $p$-form gauge theories in arbitrary spacetime dimensions $D$ (these theories are always abelian for $p>1$ ). More precisely, an 'electric' $p$-form potential $A_{\mu_{1} \ldots \mu_{p}}$ is dual to a 'magnetic' $(D-p-2)$ potential $\tilde{A}_{\mu_{1} \ldots \mu_{D-p-2}}$. A prominent example is the 3 -form potential of $D=11$ supergravity, ${ }^{19}$ with a dual 6 -form magnetic potential. Upon quantization, the duality becomes a symmetry relating the weak and strong coupling regimes by virtue of the Dirac quantization condition $e g=2 \pi i \hbar$. This is one of the reasons why such dualities have recently acquired such an importance in string theory, and why they are thought to provide an inroad into the non-perturbative structure of the theory.

Does there exist a similar symmetry for Einstein's equations? Remarkably, for linearized Einstein's equations in arbitrary space-time dimension $D$ the answer is yes. ${ }^{39,40,41,32,25,42,43}$ However, this answer will already illustrate the difficulties one encounters when one tries to extend this symmetry to the full theory. To exhibit it, let us expand the metric as $g_{\mu \nu}=\eta_{\mu \nu}+h_{\mu \nu}$, where $\eta_{\mu \nu}$ is the Minkowski metric ${ }^{\mathrm{a}}$, and the linearized fluctuations $h_{\mu \nu}$ are assumed to be small so we can neglect higher order terms. The linearized Riemann tensor is

$$
R_{\mu \nu \rho \sigma}^{L}(h)=\partial_{\mu} \partial_{\rho} h_{\nu \sigma}-\partial_{\nu} \partial_{\rho} h_{\mu \sigma}-\partial_{\mu} \partial_{\sigma} h_{\nu \rho}+\partial_{\nu} \partial_{\sigma} h_{\mu \rho}
$$

The linearized Einstein equations therefore read

$$
R_{\mu \nu}^{L}(h)=\partial^{\rho} \partial_{\rho} h_{\mu \nu}-\partial_{\mu} \partial^{\rho} h_{\rho \nu}-\partial_{\nu} \partial^{\rho} h_{\rho \mu}+\partial_{\mu} \partial_{\nu} h_{\rho}^{\rho}=0
$$

where indices are raised and lowered by means of the flat background metric $\eta^{\mu \nu}$. To reformulate thes equations in analogy with the Maxwell equations in such a way that $R_{\mu \nu}^{L}=0$ gets interchanged with a Bianchi identity, we define

$$
C_{\mu \nu \mid \rho}:=\partial_{\mu} h_{\nu \rho}-\partial_{\nu} h_{\mu \rho}
$$

This 'field strength' is of first order in the derivatives like the Maxwell field strength above, but it is not invariant under the linearized coordinate

\footnotetext{
${ }^{\text {a } I t ~ i s ~ n o t e w o r t h y ~ t h a t ~ t h e ~ c o n s t r u c t i o n ~ g i v e n ~ b e l o w ~ a p p e a r s ~ t o ~ w o r k ~ o n l y ~ f o r ~ a ~ f l a t ~}$ Minkowskian background.
} 
transformations

$$
\delta h_{\mu \nu}=\partial_{\mu} \xi_{\nu}+\partial_{\nu} \xi_{\mu} \quad \Longrightarrow \quad \delta C_{\mu \nu \mid \rho}=\partial_{\rho}\left(\partial_{\mu} \xi_{\nu}-\partial_{\nu} \xi_{\mu}\right) \neq 0
$$

This is a first difficulty: unlike ordinary gauge theories, Einstein's theory needs two derivatives for gauge covariance.

The 'Bianchi identity' now reads

$$
\partial_{[\mu} C_{\nu \rho] \mid \sigma}=0 \quad ;
$$

and is obviously different from the usual Bianchi identity on the Riemann tensor $R_{[\mu \nu \rho] \sigma}^{L}=0$. The linearized Einstein equations are now recovered from the equation of motion

$$
\partial^{\mu} C_{\mu \nu \mid \rho}=0
$$

if we impose the gauge condition

$$
C_{\mu \nu}{ }^{\nu}=0
$$

(imposing this condition is possible precisely because $C_{\mu \nu \mid \rho}$ is not gauge invariant). Noticing that (2.10) and (2.11) are completely analogous to Maxwell's equations, we now introduce the 'dual field strength'

$$
\tilde{C}_{\mu_{1} \ldots \mu_{D-2} \mid \nu}=\varepsilon_{\mu_{1} \ldots \mu_{D-2}}{ }^{\rho \sigma} C_{\rho \sigma \mid \nu}
$$

It is then easy to see that vanishing divergence for one of the field strengths implies vanishing curl for the other, and vice versa. Furthermore,

$$
\tilde{C}_{\left[\mu_{1} \ldots \mu_{D-2} \mid \nu\right]}=0 \Longleftrightarrow C_{\mu \nu}{ }^{\nu}=0
$$

On shell, where $\partial_{\left[\mu_{1}\right.} \tilde{C}_{\left.\mu_{2} \ldots \mu_{D-2}\right] \mid \nu}=0$, we can therefore introduce a 'dual graviton field' $\tilde{h}_{\mu_{1} \ldots \mu_{D-3} \mid \nu}$, analogous to the dual 'magnetic' potential $\tilde{A}_{\mu}$, with associated 'field strength'

$$
\tilde{C}_{\mu_{1} \ldots \mu_{D-2} \mid \nu}:=\partial_{\left[\mu_{1}\right.} \tilde{h}_{\left.\mu_{2} \ldots \mu_{D-2}\right] \mid \nu}
$$

Let us stress that this dual 'field strength' exists only on-shell, i.e. when the linearized Einstein equations are satisfied. The tracelessness condition (2.14) requires

$$
\tilde{h}_{\left[\mu_{1} \ldots \mu_{D-3} \mid \nu\right]}=0
$$

This is a second new feature vis-à-vis Maxwell and $p$-form gauge theories: for $D \geq 5$, the dual graviton field transforms in a mixed Young tableau representation. The associated gauge transformations are also more involved, as the gauge parameters may likewise belong to non-trivial representations. ${ }^{42}$ 
It does not appear possible to extend this duality invariance to the full non-linear theory in any obvious way. A generalization does not appear to exist even at first non-trivial order beyond the linear approximation, at least not in a way that would be compatible order by order with the background Lorentz invariance of the free theory. More succinctly, the No-Go Theorem of 42 asserts that there exists no continuous deformation of the free theory with these properties. On the other hand, experience has taught us that there is no No-Go Theorem without a loophole! So we simply interpret this result as evidence that one must search in a different direction, giving up one or more of the seemingly 'natural' assumptions that went into its proof. An example how one might possibly evade these assumptions is the one-dimensional 'geodesic' $\sigma$-model over infinite dimensional cosets which will be introduced in section 6 , and which renounces manifest space-time Lorentz invariance.

\subsection{A nonlinear duality: the Geroch group}

Unlike for the free spin-2 theory discussed in the foregoing section, there does exist a version of Einstein's theory possessing a non-linear and nonlocal duality symmetry, but it suffers from a different limitation: it works only when Einstein's theory is dimensionally reduced to two space or spacetime dimensions, i.e. in the presence of two commuting Killing vectors. For definiteness, we will take the two Killing vectors to be spacelike, and choose coordinates such that they are (locally) given by $\partial / \partial y$ and $\partial / \partial z$ : this means that the symmetry acts on solutions depending on two of the four spacetime coordinates, namely $(t, x)$. In a suitable gauge we can then write the line element $\mathrm{as}^{5}$

$$
d s^{2}=\Delta^{-1} \lambda^{2}\left(-d t^{2}+d x^{2}\right)+\left(\rho^{2} \Delta^{-1}+\Delta \tilde{B}^{2}\right) d y^{2}+2 \Delta \tilde{B} d y d z+\Delta d z^{2},
$$

where the metric coefficients depend only on $(t, x)$. The metric coefficient $\tilde{B}$ is the third component of the Kaluza Klein vector field $\left(B_{\mu}, B_{2}\right) \equiv(0,0, \tilde{B})$ that would arise in the reduction of Einstein's theory to three dimensions. The metric ansatz (2.17) can be further simplified by switching to Weyl canonical coordinates where $\rho$ is identified with the time coordinate

$$
\rho(t, x)=t
$$

This particular choice is adapted to cosmological solutions, where $t \geq 0$ with the singularity 'occurring' on the spacelike hypersurface at time $t=0$. 
This is also the physical context in which we will consider the gravitational billiards in the following section. ${ }^{\mathrm{b}}$

Here we will not write out the complete Einstein equations for the metric ansatz (2.17) (see, however, ${ }^{5,9,11}$ ) but simply note that upon dimensional reduction, the fields $(\Delta, \tilde{B})$ with $\Delta \geq 0$ coordinatize a homogeneous $\sigma$ model manifold $S L(2, \mathbb{R}) / S O(2) .{ }^{44}$ The equation for $\tilde{B}$ reads

$$
\partial_{\mu}\left(t^{-1} \Delta^{2} \partial^{\mu} \tilde{B}\right)=0
$$

with the convention, in this subsection only, that $\mu, \nu=0,1$. Because in two dimensions, every divergence-free vector field can be (locally) rewritten as a curl, we can introduce the dual 'Ehlers potential' $B(t, x)$ by means of

$$
t \Delta^{-2} \partial_{\mu} B=\epsilon_{\mu \nu} \partial^{\nu} \tilde{B}
$$

The Ehlers potential obeys the equation of motion

$$
\partial_{\mu}\left(t \Delta^{-2} \partial^{\mu} B\right)=0
$$

The combined equations of motion for $\Delta$ and $B$ can be compactly assembled into the so-called Ernst equation ${ }^{5}$

$$
\Delta \partial_{\mu}\left(t \partial^{\mu} \mathcal{E}\right)=t \partial_{\mu} \mathcal{E} \partial^{\mu} \mathcal{E}
$$

for the complex Ernst potential $\mathcal{E}:=\Delta+i B$. The pair $(\Delta, B)$ again parametrizes a coset space $S L(2, \mathbb{R}) / S O(2)$, but different from the previous one.

To write out the non-linear action of the two $S L(2, \mathbb{R})$ symmetries, one of which is the Ehlers symmetry, we use a notation that is already adapted to the Kac Moody theory in the following chapters. The relation to the more familiar 'physicist's notation' for the $S L(2, \mathbb{R})$ generators is given below:

$$
e \sim J^{+}, \quad f \sim J^{-}, \quad h \sim J^{3}
$$

In writing the variations of the fields, we will omit the infinitesimal parameter that accompanies each transformation. The Ehlers group is generated by 9,45

$$
\begin{array}{rll}
e_{3}(\Delta)=0 & , \quad e_{3}(B)=-1 \\
h_{3}(\Delta)=-2 \Delta & , \quad h_{3}(B)=-2 B \\
f_{3}(\Delta)=2 \Delta B & , \quad f_{3}(B)=B^{2}-\Delta^{2}
\end{array}
$$

\footnotetext{
${ }^{\mathrm{b}}$ If the Weyl coordinate $\rho$ is taken to be spacelike, we would be dealing with a generalization of the so-called Einstein-Rosen waves.
} 
The other $S L(2, \mathbb{R})$, often referred to as the Matzner Misner group, is generated by

$$
\begin{array}{rll}
e_{2}(\Delta)=0 & , \quad e_{2}(\tilde{B})=-1 \\
h_{2}(\Delta)=2 \Delta & , \quad h_{2}(\tilde{B})=-2 \tilde{B} \\
f_{2}(\Delta)=-2 \Delta \tilde{B} & , \quad f_{2}(\tilde{B})=\tilde{B}^{2}-\frac{\rho^{2}}{\Delta^{2}}
\end{array}
$$

(the numbering of the generators has been introduced in accordance with the numbering that will be used later in section 5). The Geroch group is now obtained by intertwining the two $S L(2, \mathbb{R})$ groups, that is by letting the Ehlers group act on $\tilde{B}$, and the Matzner Misner group on $B$, and by iterating this procedure on the resulting 'dual potentials'. It is not difficult to see that, in this process, one 'never comes back' to the original fields, and an infinite tower of dual potentials is generated. ${ }^{3}$ The Geroch group is then realized on this infinite tower; when projecting down this action onto the original fields, one ends up with a non-linear and non-local realization of this group.

The mathematical proof that the Lie algebra underlying the Geroch group is indeed $A_{1}^{(1)} \equiv \widehat{\mathfrak{s}(2, \mathbb{R})_{c e}}$ proceeds by verification of the bilinear relations (no summation on $j$ ) 45,46

$$
\left[e_{i}, f_{j}\right]=\delta_{i j} h_{j}, \quad\left[h_{i}, e_{j}\right]=A_{i j} e_{j}, \quad\left[h_{i}, f_{j}\right]=-A_{i j} f_{j}
$$

for $i, j=2,3$, with the (Cartan) matrix

$$
A_{i j}=\left(\begin{array}{cc}
2 & -2 \\
-2 & 2
\end{array}\right)
$$

The subscript ' $c e$ ' on $\widehat{\mathfrak{s}(\widehat{2, \mathbb{R}})} c$ is explained by the existence of a central extension of the loop algebra, with the central charge generator

$$
c:=h_{2}+h_{3}
$$

This charge acts on the conformal factor $\lambda$ as a scaling operator, but leaves all other fields inert ${ }^{8,9,11}$. Finally, the trilinear Serre relations

$$
\left[f_{2},\left[f_{2},\left[f_{2}, f_{3}\right]\right]\right]=\left[f_{3},\left[f_{3},\left[f_{3}, f_{2}\right]\right]\right]=0
$$

are satisfied on all fields (the corresponding relations for the $e$ generators are trivially fulfilled). Together, (2.26) and (2.29) are just the defining relations (Chevalley Serre presentation ${ }^{15,16,17}$ ) for the affine Lie algebra $A_{1}^{(1)}$.

Evidently, the relation (2.20) between $\tilde{B}$ and the Ehlers potential $B$ is a nonlinear extension of the duality

$$
\partial_{\mu} \varphi=\epsilon_{\mu \nu} \partial^{\nu} \tilde{\varphi}
$$


valid for free scalar fields in two dimensions. The main difference is that, whereas in the free field case, and more generally for $p$-form gauge theories in higher dimensions, a second dualization brings us back to the field from which we started (modulo integration constants), iterating the duality transformations (2.24) and (2.25) does not, as we pointed out already. It is therefore the intrinsic non-linearity of Einstein's theory that explains the emergence of an infinite chain of dualizations, and consequently of an infinite dimensional symmetry.

\section{Gravitational Billiards and Kac-Moody Algebras}

The duality transformations reviewed in the previous section are invariances of mutilated versions of Einstein's theory only. On the other hand, what we are really after, are symmetries that would not require any such truncations. The symmetries we are about to discuss next considerably extend the ones discussed so far, but have not actually been shown to be symmetries of Einstein's theory, or some extension thereof. There are two reasons for this. First, the full gravitational field equations are far more complicated than the truncations discussed in the foregoing section - as evidenced by the circumstance that no exact solutions appear to be known that would not make use of some kind of symmetry reduction in one way or another (in the appropriate coordinates). Consequently, any extension of the known symmetries to the full theory, which by necessity would be very non-local, will not be easy to identify. The second difficulty is that the Lie algebras that are conjectured to arise in this symmetry extension belong to the class of indefinite Kac Moody algebras. However, after more than 35 years of research in the theory of Kac Moody algebras, we still do not know much more about these algebras beyond their mere existence - despite the fact that they can be characterized by means of a simple set of generators and relations! ${ }^{\mathrm{c}}$ The main encouragement therefore derives from the fact that there exists this link between these two seemingly unrelated areas, which provides more than just a hint of an as yet undiscovered symmetry of Einstein's theory. A key role in deriving these results was played by an analysis of Einstein's equations near a spacelike singularity in terms of gravitational billiards, to which we turn next.

\footnotetext{
${ }^{\mathrm{c}}$ See remarks after table 1.1 to appreciate the challenge.
} 


\subsection{BKL dynamics and gravitational billiards}

A remarkable and most important development in theoretical cosmology was the analysis of spacelike (cosmological) singularities in Einstein's theory by Belinskii, Khalatnikov and Lifshitz (abbreviated as 'BKL' in the remainder), and their discovery of chaotic oscillations of the spacetime metric near the initial singular hypersurface; ${ }^{47}$ see also ${ }^{48,49,50,51,52}$. There is a large body of work on BKL cosmology, see ${ }^{53,54,55}$ for recent reviews and extensions of the original BKL results. In particular, there is now convincing evidence for the correctness of the basic BKL picture both from numerical analyses (see e.g. ${ }^{56,57}$ ) as well as from more rigorous work ${ }^{58,59,60,61}$. It has also been known for a long time that the chaotic oscillations of the metric near the singularity can be understood in terms of gravitational billiards, although there exist several different realizations of this description, cf. ${ }^{51,54,55}$ and references therein. The one which we will adopt here, grew out of an attempt to extend the original BKL results to more general matter coupled systems, in particular those arising in superstring and $\mathrm{M}$ theory ${ }^{62,63,24,64,65}$. It is particularly well suited for describing the relation between the BKL analysis and the theory of indefinite Kac Moody algebras, which is our main focus here, and which we will explain in the following section. See also ${ }^{66,67}$ for an alternative approach.

We first summarize the basic picture, see ${ }^{65}$ for a more detailed exposition. Our discussion will be mostly heuristic, and we shall make no attempt at rigorous proofs here (in fact, the BKL hypothesis has been rigorously proven only with very restrictive assumptions $59,57,60,61,68$, but there is so far no proof of it in the general case). Quite generally, one considers a bigbang-like space-time with an initial singular spacelike hypersurface 'located' at time $t=0$. It is then convenient to adopt a pseudo-Gaussian gauge for the metric (we will leave the number of spatial dimensions $d$ arbitrary for the moment)

$$
d s^{2}=-N^{2} d t^{2}+g_{i j} d x^{i} d x^{j}
$$

and to parametrize the spatial metric $g_{i j}$ in terms of a frame field, or dreibein, $\theta^{a}$ (a one form) ${ }^{\mathrm{d}}$

$$
g_{i j} d x^{i} \otimes d x^{j}=\sum_{a=1}^{d} \theta^{a} \otimes \theta^{a}
$$

\footnotetext{
${ }^{\mathrm{d}}$ The summation convention is in force for the coordinate indices $i, j, \ldots$, but suspended for frame indices $a, b, \ldots$.
} 
For this frame field we adopt the so-called Iwasawa decomposition

$$
\theta^{a}=e^{-\beta^{a}} \mathcal{N}^{a}{ }_{i} d x^{i}
$$

by splitting off the (logarithmic) scale factors $\beta^{a}$ from the off-diagonal frame (and metric) degrees of freedom $\mathcal{N}^{a}{ }_{i}$, which are represented by an upper triangular matrix with 1's on the diagonal. The spatial metric then becomes

$$
g_{i j}=\sum_{a=1}^{d} e^{-2 \beta^{a}} \mathcal{N}^{a}{ }_{i} \mathcal{N}^{a}{ }_{j}
$$

The main advantage of the Iwasawa decomposition is that it matches precisely with the triangular decomposition (4.48) below, which is valid for any Kac Moody algebra. Furthermore, it turns out that, in the limit $t \rightarrow 0$ all the interesting action takes place in the scale factors $\beta^{a}$, whereas the $\mathcal{N}$ as well as the matter degrees of freedom asymptotically 'come to rest' in this limit. Similarly, the metric and other degrees of freedom at different spatial points should decouple in this limit, as the spatial distance between them exceeds their horizon ${ }^{\mathrm{e}}$. The basic hypothesis underlying the BKL analysis is therefore that spatial gradients should become less and less important in comparison with time derivatives as $t \rightarrow 0$, such that the resulting theory should be effectively describable in terms of a one dimensional reduction, in which the complicated partial differential equations of Einstein's theory are effectively replaced by a continuous infinity of ordinary differential equations.

To spell out this idea in more detail, let us insert the above metric ansatz into the Einstein-Hilbert action, and drop all spatial derivatives (gradients), so that this action is approximated by a continuous superposition of onedimensional systems. One then obtains (still in $d$ spatial dimensions)

$$
S\left[g_{i j}\right]=\frac{1}{4} \int d^{d} x \int d x^{0} \tilde{N}^{-1}\left[\left(\operatorname{tr}\left(\mathrm{g}^{-1} \dot{\mathrm{g}}\right)^{2}-\left(\operatorname{trg}^{-1} \dot{\mathrm{g}}\right)^{2}\right)\right]
$$

in a matrix notation where $\mathrm{g}(t) \in G L(d, \mathbb{R})$ stands for the matrix $\left(g_{i j}\right)$ representing the spatial components of the metric at each spatial point, and $\tilde{N} \equiv N \sqrt{g}$ is a rescaled lapse function. Neglecting the off-diagonal

\footnotetext{
${ }^{\mathrm{e}}$ One might even view this decoupling as a direct consequence of the spacelike nature of the singularity.
} 
degrees of freedom, this action is further simplified to

$$
\begin{aligned}
S\left[\beta^{a}\right] & =\frac{1}{4} \int d^{d} x \int d x^{0} \tilde{N}^{-1}\left[\sum_{a=1}^{d}\left(\dot{\beta}^{a}\right)^{2}-\left(\sum_{a=1}^{d} \dot{\beta}^{a}\right)^{2}\right] \\
& \equiv \frac{1}{4} \int d^{d} x \int d x^{0} \tilde{N}^{-1} G_{a b} \dot{\beta}^{a} \dot{\beta}^{b}
\end{aligned}
$$

where $G_{a b}$ is the restriction of the superspace metric ( $\grave{a} l a$ Wheeler-DeWitt) to the space of scale factors. A remarkable, and well known property of this metric is its indefinite signature $(-+\cdots+)$, with the negative sign corresponding to variations of the conformal factor. This indefiniteness will be crucial here, because it directly relates to the indefiniteness of the generalized Cartan-Killing metric on the associated Kac Moody algebra. In the Hamiltonian description the velocities $\dot{\beta}^{a}$ are replaced by their associated momenta $\pi_{a}$; variation of the lapse $\tilde{N}$ yields the Hamiltonian constraint

$$
\mathcal{H}=\sum_{a} \pi_{a}^{2}-\frac{1}{d-1}\left(\sum_{a} \pi_{a}\right)^{2} \equiv G^{a b} \pi_{a} \pi_{b} \approx 0
$$

Here $G^{a b}$ is the inverse of the superspace metric, i.e. $G^{a c} G_{b c}=\delta_{c}^{a}$. The constraint (3.37) is supposed to hold at each spatial point, but let us concentrate at one particular spatial point for the moment. It is easy to check that (3.37) is solved by the well known conditions on the Kasner exponents. In this approximation, one thus has a Kasner-like metric at each spatial point, with the Kasner exponents depending on the spatial coordinate. In terms of the $\beta$-space description, we thus have the following picture of the dynamics of the scale factors at each spatial point. The solution to the constraint (3.37) corresponds to the motion of a relativistic massless particle (often referred to as the 'billiard ball' in the remainder) moving in the forward lightcone in $\beta$-space along a lightlike line w.r.t. the 'superspace metric' $G_{a b}$. The Hamiltonian constraint (3.37) is then re-interpreted as a relativistic dispersion relation for the 'billiard ball'.

Of course, the above approximation does not solve the Einstein equations, unless the Kasner exponents are taken to be constant (yielding the well known Kasner solution). Therefore, in a second step one must now take into account the spatial dependence and the effects of non-vanishing spatial curvature, and, eventually, the effect of matter couplings. At first sight this would seem to bring back the full complications of Einstein's equations. Surprisingly, this is not the case. Namely, one can show (at least heuristically) that ${ }^{65}$ 
(1) except for a finite number of them, the infinite number of degrees of freedom encoded in the spatially inhomogeneous metric, and in other fields, freeze in that they tend to finite limits as $t \rightarrow 0$; and

(2) the dynamics of the remaining 'active' diagonal metric degrees of freedom can be asymptotically described in terms of a simple billiard dynamics taking place in the $\beta$-space of (logarithmic) scale factors.

This result can be expressed more mathematically as follows. In the limit $t \rightarrow 0$, the effect of the remaining degrees of freedom consists simply in modifying the gravitational Hamiltonian (3.37) at a given spatial point by the addition of an effective potential that may be pictured as arising from 'integrating out' all but the diagonal degrees of freedom. Accordingly, the free Hamiltonian constraint (3.37) is now replaced by an effective Hamiltonian constraint

$$
\mathcal{H}\left(\beta^{a}, \pi_{a}, Q, P\right)=G^{a b} \pi_{a} \pi_{b}+\sum_{A} c_{A}(Q, P) \exp \left(-2 w_{A}(\beta)\right)
$$

where $\beta^{a}, \pi_{a}$ are the canonical variables corresponding to the diagonal metric degrees of freedom, and $Q, P$ denote the remaining canonical degrees of freedom. The quantities $w_{A}$ appearing in the exponential are generically linear forms in $\beta$,

$$
w_{A}(\beta)=\sum_{a}\left(w_{A}\right)_{a} \beta^{a}
$$

and are usually referred to as 'wall forms'. It is crucial that the precise form of the coefficient functions $c_{A}(Q, P)$ - which is very complicated does not matter in the BKL limit, which is furthermore dominated by a finite number of leading contributions for which $c_{A^{\prime}}(Q, P) \geq 0$. The detailed analysis ${ }^{65}$ reveals various different kinds of walls: gravitational walls due to the effect of spatial curvature, symmetry (or centrifugal) walls resulting from the elimination of off-diagonal metric components, electric and magnetic $p$-form walls, and dilaton walls. It is another non-trivial result that all these walls are timelike in $\beta$-space, that is, they have spacelike normal vectors.

The emergence of dominant walls is a consequence of the fact that, in the limit $t \rightarrow 0$, when $\beta \rightarrow \infty$, most of the walls 'disappear from sight', as the 'soft' exponential walls become steeper and steeper, eventually rising to infinity. Perhaps a useful analogy here is to think of a mountainscape, defined by the sum of the exponential potentials $C_{A} e^{-2 w_{A}}$; when the mountaintops rise into the sky, only the nearest mountains remain visible to the 
observer in the valley. The Hamiltonian constraint (3.38) then takes the limiting form

$$
H_{\infty}\left(\beta^{a}, \pi_{a}\right)=G^{a b} \pi_{a} \pi_{b}+\sum_{A^{\prime}} \Theta_{\infty}\left(-2 w_{A^{\prime}}(\beta)\right)
$$

where the sum is only over the dominant walls (indexed by $A^{\prime}$ ), and $\Theta_{\infty}$ denotes the infinite step function

$$
\Theta_{\infty}(x):= \begin{cases}0 & \text { if } x<0 \\ +\infty & \text { if } x>0\end{cases}
$$

In conclusion, the original Hamiltonian simplifies dramatically in the BKL limit $t \rightarrow 0$. The dynamics of (3.40) is still that of a massless relativistic particle in $\beta$-space, but one that is confined in a 'box'. Hence, this particle undergoes occasional collisions with the 'sharp' walls: when the argument of the $\Theta_{\infty}$ function is negative, i.e. between the walls, this particle follows a free relativistic motion characterized by the appropriate Kasner coefficients; when the particle hits a walls (where $\Theta_{\infty}$ jumps by an infinite amount), it gets reflected with a corresponding change in the Kasner exponents (these reflections are also referred to as Kasner bounces). Because the walls are timelike, the Kasner exponents get rotated by an element of the orthochronous Lorentz group in $\beta$-space at each collision.

In summary, we are indeed dealing with a relativistic billiard evolving in the forward lightcone in $\beta$-space. The billiard walls ('cushions') are the hyperplanes in $\beta$-space determined by the zeros of the wall forms, i.e. $w_{A^{\prime}}(\beta)=0$. The chamber, in which the motion takes place, is therefore the wedge-like region defined by the inequalities ${ }^{\mathrm{f}}$.

$$
w_{A^{\prime}}(\beta) \geq 0
$$

As for the long term (large $\beta$ ) behavior of the billiard, there are two possibilities: ${ }^{64}$

(1) The chamber characterized by (3.42) is entirely contained in the forward lightcone in $\beta$-space (usually with at least one edge on the lightcone). In this case, the billiard ball will undergo infinitely many collisions because, moving at the speed of light, it will always catch up with one of the walls. The corresponding metric will then exhibit infinitely

\footnotetext{
${ }^{\mathrm{f}}$ Note that this is a space-time picture in $\beta$-space: the walls recede as $t$ tends to 0 , and $\beta \rightarrow \infty$. The actual 'billiard table' can be defined as the projection of this wedge onto the unit hyperboloid $G_{a b} \beta^{a} \beta^{b}=-1$ in $\beta$-space. ${ }^{65}$ See also ${ }^{53,54,55}$ for previous work and alternative descriptions of the billiard.
} 
many Kasner bounces between $0<t<\epsilon$ for any $\epsilon>0$, hence chaotic oscillations. ${ }^{\mathrm{g}}$

(2) The chamber extends beyond the lightcone, because some walls intersect outside the lightcone. In this case the billiard ball undergoes finitely many oscillations until its motion is directed towards a region that lies outside the lightcone; it then never catches up with any wall anymore because no 'cushion' impedes its motion. The corresponding metrics therefore exhibit a monotonic Kasner-like behavior for $0<t<\epsilon$ for sufficiently small $\epsilon>0$.

The question of chaotic vs. regular behavior of the metric near the singularity is thereby reduced to determining whether the billiard chamber realizes case 1 or case 2 , and this is now a matter of a simple algebraic computation. In the case of monotonic Kasner-like behavior one can exploit these results and prove rigorous theorems about the behavior of the solution near the singularity. ${ }^{60,68}$

\subsection{Emergence of Kac Moody symmetries}

The billiard description holds not only for gravity itself, but generalizes to various kinds of matter couplings extending the Einstein-Hilbert action. However, these billiards have no special regularity properties in general. In particular, the dihedral angles between the 'walls' bounding the billiard might depend on continuous couplings, and need not be integer submultiples of $\pi$. In some instances, however, the billiard can be identified with the fundamental Weyl chamber of a symmetrizable Kac Moody algebra of indefinite type ${ }^{\mathrm{h}}$, with Lorentzian signature metric. ${ }^{24,64,69}$ Such billiards are also called 'Kac Moody billiards'. Examples are pure gravity in any number of spacetime dimensions, for which the relevant $\mathrm{KM}$ algebra is $A E_{d}$, and superstring models ${ }^{24}$ for which one obtains the rank 10 algebras $E_{10}$ and $B E_{10}$, in line with earlier conjectures made in. ${ }^{70}$ Furthermore, it was understood that chaos (finite volume of the billiard) is equivalent to hyperbolicity of the underlying Kac Moody algebra. ${ }^{64}$ Further examples of the emergence of Lorentzian Kac Moody algebras can be found in. ${ }^{69}$.

\footnotetext{
${ }^{\mathrm{g}}$ Although we utilise this term in a somewhat cavalier manner here, readers can be assured that this system is indeed chaotic in the rigorous sense. For instance, projection onto the unit hyperboloid in $\beta$-space leads to a finite volume billiard on a hyperbolic manifold of constant negative curvature, which is known to be strongly chaotic.

${ }^{\text {h}}$ From now on we abbreviate 'Kac Moody' by 'KM', and 'Cartan subalgebra' by 'CSA'.
} 
The main feature of the gravitational billiards that can be associated with $\mathrm{KM}$ algebras is that there exists a group theoretical interpretation of the billiard motion: the asymptotic BKL dynamics is equivalent, at each spatial point, to the asymptotic dynamics of a one-dimensional nonlinear $\sigma$-model based on a certain infinite dimensional coset space $G / K(G)$, where the KM group $G$ and its maximal compact subgroup $K(G)$ depend on the specific model. In particular, the $\beta$-space of logarithmic scale factors, in which the billard motion takes place, can be identified with the Cartan subalgebra (CSA) of the underlying indefinite Kac-Moody algebra. The dominant walls that determine the billiards asymptotically are associated with the simple roots of the KM algebra. We emphasize that it is precisely the presence of gravity, which comes with an indefinite (Lorentzian) metric in the $\beta$-superspace, hence a Cartan-Killing metric of indefinite signature, which forces us to consider infinite dimensional KM groups. By contrast, the finite dimensional simple Lie algebras, which can also be considered as KM algebras, but which were already classified long ago by Cartan, are characterized by a positive definite Cartan-Killing metric.

The $\sigma$-model formulation to be introduced and elaborated in section 6 enables one to go beyond the BKL limit, and to see the beginnings of a possible identification of the dynamics of the scale factors and of all the remaining variables with that of a non-linear $\sigma$-model defined on the cosets of the KM group divided by its maximal compact subgroup. ${ }^{25,27}$ In that formulation, the various types of walls can thus be understood directly as arising from the large field limit of the corresponding $\sigma$-models. So far, only two examples have been considered in this context, namely pure gravity, in which case the relevant $\mathrm{KM}$ algebra is $A E_{3},{ }^{65}$ and the bosonic sector of $D=11$ supergravity, for which the relevant algebra is the maximal rank 10 hyperbolic KM algebra $E_{10}$; we will return to the latter model in the final section. Following Ref. 25, 27 one can introduce for both models a precise identification between the purely $t$-dependent $\sigma$-model quantities obtained from the geodesic action on the $G / K(G)$ coset space on the one hand, and the fields and their spatial gradients evaluated at a given, but arbitrarily chosen spatial point on the other.

\subsection{The main conjecture}

To sum up, it has been established that

(1) in many physical theories of interest (and all the models arising in supergravity and superstring theory), the billiard region in which the 
dynamics of the active degrees of freedom takes place can be identified with the Weyl chamber of some Lorentzian KM algebra; and

(2) the concept of a nonlinear $\sigma$-model on a coset space $G / K(G)$ can be generalized to the case where $G$ is a Lorentzian KM group, and $K(G)$ its 'maximal compact subgroup'; furthermore, these (one-dimensional) $\sigma$-models are asymptotically equivalent to the billiard dynamics describing the active degrees of freedom as $t \rightarrow 0$.

So far, these correspondences between gravity or supergravity models on the one hand, and certain KM coset space $\sigma$-models on the other, work only for truncated versions of both models. Namely, on the gravity side one has to restrict the dependence on the spatial coordinates, whereas the KM models must be analyzed in terms of a 'level expansion', in which only the lowest orders are retained, and the remaining vast expanse of the KM Lie algebra remains to be understood and explored. There are, however, indications that, at least as far as the higher order spatial gradients on the (super)gravity side are concerned, the correspondence can be further extended: the level expansions of $A E_{3}$, and other hyperbolic KM algebras contain all the requisite representations needed for the higher order spatial gradients $^{25}$ (as well as an exponentially increasing number of representations for which a physical interpretation remains to be found ${ }^{71}$ ). This observation gave rise to the key conjecture ${ }^{25}$ for the correspondence between $D=11$ supergravity and the $E_{10} / K\left(E_{10}\right)$ coset model, which we here reformulate in a somewhat more general manner:

The time evolution of the geometric data at each spatial point, i.e. the values of all the fields and their spatial gradients, can be mapped onto some constrained null geodesic motion on the infinite dimensional $G / K(G)$ coset space.

If true, this conjecture would provide us with an entirely new way of describing and analyzing a set of (non-linear) partial differential equations in terms of an ordinary differential equation in infinitely many variables, by 'spreading' the spatial dependence over an infinite dimensional Lie algebra, and thereby mapping the cosmological evolution onto a single trajectory in the corresponding coset space. In the remainder of this article we will therefore spell out some of the technical details that lead up to this conjecture. 


\section{Basics of Kac Moody theory}

We here summarize some basic results from the theory of KM algebras, referring the reader to ${ }^{15,16,17}$ for comprehensive treatments. Every KM algebra $\mathfrak{g} \equiv \mathfrak{g}(A)$ can be defined by means of an integer-valued Cartan matrix $A$ and a set of generators and relations. We shall assume that the Cartan matrix is symmetrizable since this is the case encountered for cosmological billiards. The Cartan matrix can then be written as $(i, j=1, \ldots r$, with $r$ denoting the rank of $\mathfrak{g}(A)$ )

$$
A_{i j}=\frac{2\left\langle\alpha_{i} \mid \alpha_{j}\right\rangle}{\left\langle\alpha_{i} \mid \alpha_{i}\right\rangle}
$$

where $\left\{\alpha_{i}\right\}$ is a set of $r$ simple roots, and where the angular brackets denote the invariant symmetric bilinear form of $\mathfrak{g}(A) .{ }^{15}$ Recall that the roots can be abstractly defined as linear forms on the Cartan subalgebra (CSA) $\mathfrak{h} \subset \mathfrak{g}(A)$. The generators, which are also referred to as Chevalley-Serre generators, consist of triples $\left\{h_{i}, e_{i}, f_{i}\right\}$ with $i=1, \ldots, r$, and for each $i$ form an $\mathfrak{s l}(2, \mathbb{R})$ subalgebra. The CSA $\mathfrak{h}$ is then spanned by the elements $h_{i}$, so that

$$
\left[h_{i}, h_{j}\right]=0
$$

The remaining relations generalize the ones we already encountered in Eqs. (2.26) and (2.29): Furthermore,

$$
\left[e_{i}, f_{j}\right]=\delta_{i j} h_{j}
$$

and

$$
\left[h_{i}, e_{j}\right]=A_{i j} e_{j}, \quad\left[h_{i}, f_{j}\right]=-A_{i j} f_{j}
$$

so that the value of the linear form $\alpha_{j}$, corresponding to the raising operator $e_{j}$, on the element $h_{i}$ of the preferred basis $\left\{h_{i}\right\}$ of $\mathfrak{h}$ is $\alpha_{j}\left(h_{i}\right)=A_{i j}$. More abstractly, and independently of the choice of any basis in the CSA, the roots appear as eigenvalues of the adjoint action of any element $h$ of the CSA on the raising $\left(e_{i}\right)$ or lowering $\left(f_{i}\right)$ generators: $\left[h, e_{i}\right]=+\alpha_{i}(h) e_{i}$, $\left[h, f_{i}\right]=-\alpha_{i}(h) f_{i}$. Last but not least we have the so-called Serre relations

$$
\operatorname{ad}\left(e_{i}\right)^{1-A_{i j}}\left(e_{j}\right)=0, \quad \operatorname{ad}\left(f_{i}\right)^{1-A_{i j}}\left(f_{j}\right)=0
$$

A key property of every $\mathrm{KM}$ algebra is the triangular decomposition

$$
\mathfrak{g}(A)=\mathfrak{n}^{-} \oplus \mathfrak{h} \oplus \mathfrak{n}^{+}
$$

where $\mathfrak{n}^{+}$and $\mathfrak{n}^{-}$, respectively, are spanned by the multiple commutators of the $e_{i}$ and $f_{i}$ which do not vanish on account of the Serre relations or the 
Jacobi identity. To be completely precise, $\mathfrak{n}^{+}$is the quotient of the free Lie algebra generated by the $e_{i}$ 's by the ideal generated by the Serre relations (idem for $\mathfrak{n}^{-}$and $f_{i}$ ). In more mundane terms, when the algebra is realized, in a suitable basis, by infinite dimensional matrices, $\mathfrak{n}^{+}$and $\mathfrak{n}^{-}$simply consist of the 'nilpotent' matrices with nonzero entries only above or below the diagonal. Exponentiating them formally, one obtains infinite dimensional matrices again with nonzero entries above or below the diagonal.

A main result of the general theory is that, for positive definite $A$, one just recovers from these relations Cartan's list of finite dimensional Lie algebras (see e.g. ${ }^{72}$ for an introduction). For non positive-definite $A$, on the other hand, the associated KM algebras are infinite dimensional. If $A$ has only one zero eigenvalue, with all other eigenvalues strictly positive, the associated algebra is called affine. The simplest example of such an algebra is the the $A_{1}^{(1)}$ algebra underlying the Geroch group, which we already encountered and discussed in section 2.2, with Cartan matrix (2.27). While the structure and properties of affine algebras are reasonably well understood, ${ }^{15,17}$ this is not so for indefinite $A$, when at least one eigenvalue of $A$ is negative. In this case, very little is known, and it remains an outstanding problem to find a manageable representation for them. ${ }^{15,16} \mathrm{In}$ particular, there is not a single example of an indefinite KM algebra for which the root multiplicities, i.e. the number of Lie algebra elements associated with a given root, are known in closed form. The scarcity of results is even more acute for the 'Kac-Moody groups' obtained by formal exponentiation of the associated Lie algebras. As a special, and important case, the class of Lorentzian KM algebras includes hyperbolic KM algebras whose Cartan matrices are such that the deletion of any node from the Dynkin diagram leaves either a finite or an affine subalgebra, or a disjoint union of them.

The 'maximal compact' subalgebra $\mathfrak{k}$ is defined as the invariant subalgebra of $\mathfrak{g}(A)$ under the standard Chevalley involution, i.e.

$$
\theta(x)=x \quad \text { for all } x \in \mathfrak{k}
$$

with

$$
\theta\left(h_{i}\right)=-h_{i}, \quad \theta\left(e_{i}\right)=-f_{i}, \quad \theta\left(f_{i}\right)=-e_{i}
$$

More explicitly, it is the subalgebra generated by multiple commutators of $\left(e_{i}-f_{i}\right)$. For finite dimensional $\mathfrak{g}(A)$, the inner product induced on the maximal compact subalgebra $\mathfrak{k}$ is negative-definite, and the orthogonal complement to $\mathfrak{k}$ has a positive definite inner product. This is not true, however, 
for indefinite $A$. It is sometimes convenient to introduce the operation of transposition acting on any Lie algebra element $E$ as

$$
E^{T}:=-\theta(E)
$$

The subalgebra $\mathfrak{k}$ is thus generated by the 'anti-symmetric' elements satisfying $E^{T}=-E$; after exponentiation, the elements of the maximally compact subgroup $K$ formally appear as 'orthogonal matrices' obeying $k^{T}=k^{-1}$.

Often one uses a so-called Cartan-Weyl basis for $\mathfrak{g}(A)$. Using Greek indices $\mu, \nu, \ldots$ to label the root components corresponding to an arbitrary basis $H_{\mu}$ in the CSA, with the usual summation convention and a Lorentzian metric $G_{\mu \nu}$ for an indefinite $\mathfrak{g}$, we have $h_{i}:=\alpha_{i}^{\mu} H_{\mu}$, where $\alpha_{i}^{\mu}$ are the 'contravariant components', $G_{\mu \nu} \alpha_{i}^{\nu} \equiv \alpha_{i \mu}$, of the simple roots $\alpha_{i}$ $(i=1, \ldots r)$, which are linear forms on the CSA, with 'covariant components' defined as $\alpha_{i \mu} \equiv \alpha_{i}\left(H_{\mu}\right)$. To an arbitrary root $\alpha$ there corresponds a set of Lie-algebra generators $E_{\alpha, s}$, where $s=1, \ldots$, mult $(\alpha)$ labels the (in general) multiple Lie-algebra elements associated with $\alpha$. The root multiplicity mult $(\alpha)$ is always one for finite dimensional Lie algebras, and also for the real (= positive norm) roots, but generically grows exponentiallly with $-\alpha^{2}$ for indefinite $A$. In this notation, the remaining Chevalley-Serre generators are given by $e_{i}:=E_{\alpha_{i}}$ and $f_{i}:=E_{-\alpha_{i}}$. Then,

$$
\left[H_{\mu}, E_{\alpha, s}\right]=\alpha_{\mu} E_{\alpha, s}
$$

and

$$
\left[E_{\alpha, s}, E_{\alpha^{\prime}, t}\right]=\sum_{u} c_{\alpha \alpha^{\prime}}^{s, t, u} E_{\alpha+\alpha^{\prime}, u}
$$

The elements of the Cartan-Weyl basis are normalized such that

$$
\left\langle H_{\mu} \mid H_{\nu}\right\rangle=G_{\mu \nu}, \quad\left\langle E_{\alpha, s} \mid E_{\beta, t}\right\rangle=\delta_{s t} \delta_{\alpha+\beta, 0}
$$

where we have assumed that the basis satisfies $E_{\alpha, s}^{T}=E_{-\alpha, s}$. Let us finally recall that the Weyl group of a KM algebra is the discrete group generated by reflections in the hyperplanes orthogonal to the simple roots.

\section{The hyperbolic Kac Moody algebra $A E_{3}$}

As we explained, the known symmetries of Einstein's theory for special types of solutions include the Ehlers and Matzner Misner $S L(2, \mathbb{R})$ symmetries, which can be combined into the Geroch group $S \widehat{L(2, \mathbb{R})})_{c e}$. Furthermore, in the reduction to one time dimension, Einstein's theory is invariant under a rigid $S L(3, \mathbb{R})$ symmetry acting on the spatial dreibein. Hence, any 
conjectured symmetry of Einstein's theory should therefore contain these symmetries as subgroups. Remarkably, there is a hyperbolic KM group with precisely these properties, whose Lie algebra is furthermore the simplest hyperbolic KM algebra containing an affine subalgebra. ${ }^{46}$ This is the algebra $A E_{3}$, with Cartan matrix

$$
A_{i j}=\left(\begin{array}{ccc}
2 & -1 & 0 \\
-1 & 2 & -2 \\
0 & -2 & 2
\end{array}\right)
$$

The $\mathfrak{s l}(2, \mathbb{R})$ subalgebra corresponding to the third diagonal entry of $A_{i j}$ is associated with the Ehlers group. The affine subgroup corresponding to the submatrix (2.27) is the Geroch group ${ }^{3}$ already discussed in section 2.2. The $S L(3, \mathbb{R})$ subgroup containing the the Matzner-Misner $S L(2, \mathbb{R})$ group, is generated by $\left(e_{1}, f_{1}, h_{1}\right)$ and $\left(e_{2}, f_{2}, h_{2}\right)$, corresponding to the submatrix

$$
\left(\begin{array}{cc}
2 & -1 \\
-1 & 2
\end{array}\right)
$$

As we said, not much is known about $A E_{3}$; in particular, there is no 'list' of its (infinitely many) generators, nor of its structure constants (which are certainly too numerous to fit in any list, see below!). Nevertheless, in order to gain some 'feeling' for this algebra, we will now work out the beginnings of its decomposition into irreducible representations of its $S L(3, \mathbb{R})$ subgroup. Of course, this decomposition refers to the adjoint action of the $\mathfrak{s l}(3, \mathbb{R})$ subalgebra embedded in $A E_{3}$. More specifically, we will analyze the lowest terms of the nilpotent subalgebra $\mathfrak{n}^{+}$. To do so, we first define, for any given root $\alpha$, its $\mathfrak{s l}(3, \mathbb{R})$ level $\ell$ to be the number of times the root $\alpha_{3}$ appears in it, to wit, $\alpha=m \alpha_{1}+n \alpha_{2}+\ell \alpha_{3}$. The algebra $A E_{3}$ thereby decomposes into an infinite irreducible representations of its $\mathfrak{s t}(3, \mathbb{R})$ subalgebra ${ }^{\mathrm{i}}$. As is well known, ${ }^{72}$ the irreducible representations of $\mathfrak{s l}(3, \mathbb{R})$ can be conveniently characterized by their Dynkin labels $\left[p_{1}, p_{2}\right]$. In terms of the Young tableau description of $\mathfrak{s l}(3, \mathbb{R})$ representations, the first Dynkin label $p_{1}$ counts the number of columns having two boxes, while $p_{2}$ counts the number of columns having only one box. For instance, $\left[p_{1}, p_{2}\right]=[1,0]$ labels an antisymmetric two-index tensor, while $\left[p_{1}, p_{2}\right]=[0,2]$ denotes a symmetric two-index tensor. The dimension of the representation $\left[p_{1}, p_{2}\right]$ is $\left(p_{1}+1\right)\left(p_{2}+1\right)\left(p_{1}+p_{2}+2\right) / 2$.

\footnotetext{
${ }^{\mathrm{i}}$ A different decomposition would be one in terms of the affine subalgebra $A_{1}^{(1)} \subset A E_{3}$; 18 however, the representation theory of $A_{1}^{(1)}$ is far more complicated and much less developed than that of $\mathfrak{s l}(3, \mathbb{R})$.
} 
The level $\ell=0$ sector, which includes the third Cartan generator $h_{3}$, is the $\mathfrak{g l}(3, \mathbb{R})$ subalgebra with generators $K^{i}{ }_{j}$ (where $i, j=1,2,3$ ) and commutation relations

$$
\left[K^{i}{ }_{j}, K^{k}{ }_{l}\right]=\delta_{j}^{k} K^{i}{ }_{l}-\delta_{l}^{i} K^{k}{ }_{j}
$$

corresponding to the $G L(3, \mathbb{R})$ group acting on the spatial components of the vierbein. The restriction of the $A E_{3}$-invariant bilinear form to the level0 sector is

$$
\left\langle K^{i}{ }_{j} \mid K^{k}{ }_{l}\right\rangle=\delta_{l}^{i} \delta_{j}^{k}-\delta_{j}^{i} \delta_{l}^{k}
$$

The identification with the Chevalley-Serre generators is

$$
\begin{array}{ll}
e_{1}=K^{1}{ }_{2}, \quad f_{1}=K^{2}{ }_{1}, & h_{1}=K^{1}{ }_{1}-K^{2}{ }_{2} \\
e_{2}=K^{2}{ }_{3}, \quad f_{2}=K^{3}{ }_{2}, & h_{2}=K^{2}{ }_{2}-K^{3}{ }_{3} \\
h_{3}=-K^{1}{ }_{1}-K^{2}{ }_{2}+K^{3}{ }_{3} &
\end{array}
$$

showing how the over-extended CSA generator $h_{3}$ enlarges the original $\mathfrak{s l}(3, \mathbb{R})$ generated by $\left(e_{1}, f_{1}, h_{1}\right)$ and $\left(e_{2}, f_{2}, h_{2}\right)$ to the Lie algebra $\mathfrak{g l}(3, \mathbb{R})$. The CSA generators are related to the 'central charge' generator $c$ by

$$
c=h_{2}+h_{3}=-K^{1}
$$

which acts as a scaling on the conformal factor ${ }^{8,9,11}$ (here realized as the 1-1 component of the vierbein).

To determine the representations of $\mathfrak{s l}(3, \mathbb{R})$ appearing at levels $\ell=$ \pm 1 , we observe that, under the adjoint action of $\mathfrak{s l}(3, \mathbb{R})$, i.e. of $\left(e_{1}, f_{1}, h_{1}\right)$ and $\left(e_{2}, f_{2}, h_{2}\right)$, the extra Chevalley-Serre generator $f_{3}$ is a highest weight vector:

$$
\begin{aligned}
& e_{1}\left(f_{3}\right) \equiv\left[e_{1}, f_{3}\right]=0 \\
& e_{2}\left(f_{3}\right) \equiv\left[e_{2}, f_{3}\right]=0
\end{aligned}
$$

The Dynkin labels of the representation built on this highest weight vector $f_{3}$ are $\left(p_{1}, p_{2}\right)=(0,2)$, since

$$
\begin{aligned}
& h_{1}\left(f_{3}\right) \equiv\left[h_{1}, f_{3}\right]=0 \\
& h_{2}\left(f_{3}\right) \equiv\left[h_{2}, f_{3}\right]=2 f_{3}
\end{aligned}
$$

As mentioned above, the representation $\left(p_{1}, p_{2}\right)=(0,2)$ corresponds to a symmetric (two-index) tensor. Hence, at the levels \pm 1 we have $A E_{3}$ generators which can be represented as symmetric tensors $E^{i j}=E^{j i}$ 
and $F_{i j}=F_{j i}$. One verifies that all algebra relations are satisfied with $\left(a_{(i j)} \equiv\left(a_{i j}+a_{j i}\right) / 2\right)$

$$
\begin{aligned}
{\left[K^{i}{ }_{j}, E^{k l}\right] } & =\delta_{j}^{k} E^{i l}+\delta_{j}^{l} E^{k i} \\
{\left[K^{i}{ }_{j}, F_{k l}\right] } & =-\delta_{k}^{i} F_{j l}-\delta_{l}^{i} F_{k j} \\
{\left[E^{i j}, F_{k l}\right] } & =2 \delta_{(k}^{(i} K^{j)}{ }_{l)}-\delta_{k}^{(i} \delta_{l}^{j)}\left(K^{1}{ }_{1}+K^{2}{ }_{2}+K^{3}{ }_{3}\right) \\
\left\langle F_{i j} \mid E^{k l}\right\rangle & =\delta_{i}^{(k} \delta_{j}^{l)}
\end{aligned}
$$

and the identifications

$$
e_{3}=E^{33}, \quad f_{3}=F_{33}
$$

As one proceeds to higher levels, the classification of $\mathfrak{s l}(3, \mathbb{R})$ representations becomes rapidly more involved due to the exponential increase in the number of representations with level $\ell$. Generally, the representations that can occur at level $\ell+1$ must be contained in the product of the level- $\ell$ representations with the level-one representation $(0,2)$. Working out these products is elementary, but cumbersome. For instance, the level-two generator $E^{a b \mid j k} \equiv \varepsilon^{a b i} E_{i}{ }^{j k}$, with labels $(1,2)$, is straightforwardly obtained by commuting two level-one elements

$$
\left[E^{i j}, E^{k l}\right]=\varepsilon^{m k(i} E_{m}^{j) l}+\varepsilon^{m l(i} E_{m}^{j) k}
$$

A more economical way to identify the relevant representations is to work out the relation between Dynkin labels and the associated highest weights, using the fact that the highest weights of the adjoint representation are the roots. More precisely, the highest weight vectors being (as exemplified above at level 1) of the 'lowering type', the corresponding highest weights are negative roots, say $\Lambda=-\alpha$. Working out the associated Dynkin labels one obtains

$$
p_{1} \equiv p=n-2 m, \quad p_{2} \equiv q=2 \ell+m-2 n
$$

As indicated, we shall henceforth use the notation $\left[p_{1}, p_{2}\right] \equiv[p, q]$ for the Dynkin labels. This formula is restrictive because all the integers entering it must be non-negative. Inverting this relation we get

$$
\begin{aligned}
m & =\frac{2}{3} \ell-\frac{2}{3} p-\frac{1}{3} q \\
n & =\frac{4}{3} \ell-\frac{1}{3} p-\frac{2}{3} q
\end{aligned}
$$

with $n \geq 2 m \geq 0$. A further restriction derives from the fact that the highest weight must be a root of $A E_{3}$, viz. its square must be smaller or equal to 2 :

$$
\Lambda^{2}=\frac{2}{3}\left(p^{2}+q^{2}+p q-\ell^{2}\right) \leq 2
$$


Consequently, the representations occurring at level $\ell$ must belong to the list of all the solutions of (5.67) which are such that the labels $m, n, p, q$ are non-negative integers and the highest weight $\Lambda$ is a root, i.e. $\Lambda^{2} \leq 2$. These simple diophantine equations/inequalities can be easily evaluated by hand up to rather high levels.

Although the above procedure substantially reduces the number of possibilities, it does not tell us how often a given representation appears, i.e. its outer multiplicity $\mu$. For this purpose we have to make use of more detailed information about $A E_{3}$, namely the root multiplicities computed in. ${ }^{18,15}$ Matching the combined weight diagrams with the root multiplicities listed in table $H_{3}$ on page 215 of, ${ }^{15}$ one obtains the following representations in the decomposition of $A E_{3}$ w.r.t. its $\mathfrak{s l}(3, \mathbb{R})$ subalgebra up to level $\ell \leq 5$, where we also indicate the root coefficients $\left(m_{1}, m_{2}, \ell\right)$, the norm and multiplicity of the root $\alpha$, and the outer multiplicity of the representation $[p, q]$ :

\begin{tabular}{|c|c|c|c|c|c|}
\hline$\ell$ & {$[p, q]$} & $\alpha$ & $\overline{\alpha^{2}}$ & mult $\alpha$ & $\mu$ \\
\hline 1 & {$[0,2]$} & $(0,0,1)$ & 2 & 1 & 1 \\
\hline 2 & {$[1,2]$} & $(0,1,2)$ & 2 & 1 & 1 \\
\hline \multirow[t]{2}{*}{3} & {$[2,2]$} & $(0,2,3)$ & 2 & 1 & 1 \\
\hline & {$[1,1]$} & $(1,3,3)$ & -4 & 3 & 1 \\
\hline \multirow[t]{5}{*}{4} & {$[3,2]$} & $(0,3,4)$ & 2 & 1 & 1 \\
\hline & {$[2,1]$} & $(1,4,4)$ & -6 & 5 & 2 \\
\hline & {$[1,0]$} & $(2,5,4)$ & -10 & 11 & 1 \\
\hline & {$[0,2]$} & $(2,4,4)$ & -8 & 7 & 1 \\
\hline & {$[1,3]$} & $(1,3,4)$ & -2 & 2 & 1 \\
\hline \multirow[t]{7}{*}{5} & {$[4,2]$} & $(0,4,5)$ & 2 & 1 & 1 \\
\hline & {$[3,1]$} & $(1,5,5)$ & -8 & 7 & 3 \\
\hline & {$[2,0]$} & $(2,6,5)$ & -14 & 22 & 3 \\
\hline & {$[0,1]$} & $(3,6,5)$ & -16 & 30 & 2 \\
\hline & {$[0,4]$} & $(2,4,5)$ & -6 & 5 & 2 \\
\hline & {$[1,2]$} & $(2,5,5)$ & -12 & 15 & 4 \\
\hline & {$[2,3]$} & $(1,4,5)$ & -4 & 3 & 2 \\
\hline
\end{tabular}

Table 1.1.: Decomposition of $A E_{3}$ under $\mathfrak{s l}(3, \mathbb{R})$ for $\ell \leq 5$.

The above table does not look too bad, but appearances are deceptive, because the number of representations grows exponentially with the level! 
For $A E_{3}$, the list of representations with their outer multiplicities is meanwhile available up to $\ell \leq 56^{71}$; the total number of representations up to that level is $20994472770550672476591949725720^{\text {j }}$, larger than $10^{31}$ ! This number should suffice to convince readers of the 'explosion' that takes place in these algebras as one increases the level. Similar decompositions can be worked out for the indefinite Kac-Moody algebras $E_{10}$ and $E_{11}{ }^{71}$, and for $E_{10}$ under its $D_{9}$ and $A_{8} \times A_{1}$ subalgebras. ${ }^{26,28}$. The real problem, however, is not so much the large number of representations, but rather the absence of any discernible structure in these tables, at least up until now.

\section{Nonlinear $\sigma$-Models in one dimension}

Notwithstanding the fact that we know even less about the groups associated with indefinite $\mathrm{KM}$ algebras, it is possible to formulate nonlinear $\sigma$-models in one time dimension and thereby provide an effective and unified description of the asymptotic BKL dynamics for several physically important models. The basic object of interest is a one-parameter dependent KM group element $\mathcal{V}=\mathcal{V}(t)$, assumed to be an element of the coset space $G / K(G)$, where $G$ is the group obtained by formal exponentiation of the KM algebra $\mathfrak{g}$, and $K(G)$ its maximal compact subgroup, obtained by formal exponentiation of the associated maximal compact subalgebra $\mathfrak{k}$ defined above. For finite dimensional $\mathfrak{g}(A)$ our definitions reduce to the usual ones, whereas for indefinite KM algebras they are formal constructs to begin with. In order to ensure that our definitions are meaningful operationally, we must make sure at every step that any finite truncation of the model is well defined and can be worked out explicitly in a finite number of steps.

In physical terms, $\mathcal{V}$ can be thought of as an extension of the vielbein of general relativity, with $G$ and $K(G)$ as generalizations of the $G L(d, \mathbb{R})$ and local Lorentz symmetries of general relativity. For infinite dimensional $G$, the object $\mathcal{V}$ thus is a kind of ' $\infty$-bein', that can be associated with the 'metric'

$$
\mathcal{M}:=\mathcal{V}^{T} \mathcal{V}
$$

which is invariant under the left action $(\mathcal{V} \rightarrow k \mathcal{V})$ of the 'Lorentz group' $K(G)$. Exploiting this invariance, we can formally bring $\mathcal{V}$ into a triangular gauge

$$
\mathcal{V}=\mathcal{A} \cdot \mathcal{N} \Longrightarrow \mathcal{M}=\mathcal{N}^{T} \mathcal{A}^{2} \mathcal{N}
$$

\footnotetext{
${ }^{\mathrm{j}}$ T. Fischbacher, private communication.
} 
where the abelian part $\mathcal{A}$ belongs to the exponentiation of the CSA, and the nilpotent part $\mathcal{N}$ to the exponentiation of $\mathfrak{n}^{+}$. This formal Iwasawa decomposition, which is the infinite dimensional analog of (3.33), can be made fully explicit by decomposing $\mathcal{A}$ and $\mathcal{N}$ in terms of bases of $\mathfrak{h}$ and $\mathfrak{n}^{+}$ (using the Cartan Weyl basis)

$$
\begin{aligned}
\mathcal{A}(t) & =\exp \left(\beta^{\mu}(t) H_{\mu}\right), \\
\mathcal{N}(t) & =\exp \left(\sum_{\alpha \in \Delta_{+}} \sum_{s=1}^{\operatorname{mult}(\alpha)} \nu_{\alpha, s}(t) E_{\alpha, s}\right)
\end{aligned}
$$

where $\Delta_{+}$denotes the set of positive roots. The components $\beta^{\mu}$, parametrizing a generic element in the CSA $\mathfrak{h}$, will turn out to be in direct correspondence with the metric scale factors $\beta^{a}$ in (3.34). The main technical difference with the kind of Iwasawa decompositions used in section 3.1 is that now the matrix $\mathcal{V}(t)$ is infinite dimensional for indefinite $\mathfrak{g}(A)$, in which case the decomposition (6.71) is, in fact, the only sensible parametrization available! Consequently, there are now infinitely many $\nu$ 's, whence $\mathcal{N}$ contains an infinite tower of new degrees of freedom. Next we define

$$
\dot{\mathcal{N} \mathcal{N}^{-1}}=\sum_{\alpha \in \Delta_{+}} \sum_{s=1}^{\operatorname{mult}(\alpha)} j_{\alpha, s} E_{\alpha, s} \in \mathfrak{n}^{+}
$$

with

$$
j_{\alpha, s}=\dot{\nu}_{\alpha, s}+" \nu \dot{\nu}+\nu \nu \dot{\nu}+\cdots "
$$

(we put quotation marks to avoid having to write out the indices). To define a Lagrangian we consider the quantity

$$
\dot{\mathcal{V}} \mathcal{V}^{-1}=\dot{\beta}^{\mu} H_{\mu}+\sum_{\alpha \in \Delta_{+}} \sum_{s=1}^{\operatorname{mult}(\alpha)} \exp (\alpha(\beta)) j_{\alpha, s} E_{\alpha, s}
$$

which has values in the Lie algebra $\mathfrak{g}(A)$. Here we have set

$$
\alpha(\beta) \equiv \alpha_{\mu} \beta^{\mu}
$$

for the value of the root $\alpha$ ( $\equiv$ linear form) on the CSA element $\beta=\beta^{\mu} H_{\mu}$. Next we define

$$
\begin{aligned}
P & :=\frac{1}{2}\left(\dot{\mathcal{V}} \mathcal{V}^{-1}+\left(\dot{\mathcal{V}} \mathcal{V}^{-1}\right)^{T}\right) \\
& =\dot{\beta}^{\mu} H_{\mu}+\frac{1}{2} \sum_{\alpha \in \Delta_{+}} \sum_{s=1}^{\operatorname{mult}(\alpha)} j_{\alpha, s} \exp (\alpha(\beta))\left(E_{\alpha, s}+E_{-\alpha, s}\right)
\end{aligned}
$$


where we arranged the basis so that $E_{\alpha, s}^{T}=E_{-\alpha, s}$. The KM-invariant $\sigma$ model Lagrangian is defined by means of the KM-invariant bilinear form

$$
\begin{aligned}
\mathcal{L} & =\frac{1}{2} n^{-1}\langle P \mid P\rangle \\
& =n^{-1}\left(\frac{1}{2} G_{\mu \nu} \dot{\beta}^{\mu} \dot{\beta}^{\nu}+\frac{1}{4} \sum_{\alpha \in \Delta_{+}} \sum_{s=1}^{\operatorname{mult}(\alpha)} \exp (2 \alpha(\beta)) j_{\alpha, s} j_{\alpha, s}\right)
\end{aligned}
$$

Here the Lorentzian metric $G_{\mu \nu}$ is the restriction of the invariant bilinear form to the CSA, cf. (4.54). The 'lapse function' $n$ ensures that our formalism is invariant under reparametrizations of the time variable. Remarkably, this action defined by the above Lagrangian is essentially unique because there are no higher order polynomial invariants for indefinite KM algebras. ${ }^{15}$

After these preparations we are now ready to specialize to the algebra $A E_{3}$. In this case this Lagrangian (6.77) contains the Kasner Lagrangian (3.35) as a special truncation. More specifically, retaining only the level zero fields (corresponding to the 'sub-coset' $G L(3, \mathbb{R}) / O(3)$ )

$$
\left.\mathcal{V}(t)\right|_{\ell=0}=\exp \left(h_{b}^{a}(t) K_{a}^{b}\right)
$$

and defining from $h^{a}{ }_{b}$ a vielbein by matrix exponentiation $e^{a}{ }_{b} \equiv(\exp h)^{a}{ }_{b}$, and a corresponding contravariant metric $g^{a b}=e^{a}{ }_{c} e^{b}{ }_{c}$, it turns out that the bilinear form (5.58) reproduces the Lagrangian (3.35) (for the special case of three spatial dimensions). This means that we can identify the restriction $G_{\mu \nu}$ of the Cartan-Killing metric to the CSA with the superspace metric $G_{a b}$ in the superspace of scale factors $\beta$ in (3.35).

At level $\ell=1$, we have the fields $\phi_{i j}$ associated with the level-one generators $E^{i j}$. Observe that for $D=4$, these are precisely the spatial components of the dual graviton introduced in (2.15) - in other words, we have rederived the result of section 2.2 by a purely group theoretical argument! (This argument works likewise for $D>4$.) This leads to a slightly less restricted truncation of our KM-invariant $\sigma$-model

$$
\left.\mathcal{V}(t)\right|_{\ell=0,1}=\exp \left(h_{b}^{a}(t) K_{a}^{b}\right) \exp \left(\phi_{a b} E^{a b}\right)
$$

In the gauge $n=1$, the Lagrangian now has the form $\mathcal{L} \sim\left(g^{-1} \dot{g}\right)^{2}+$ $g^{-1} g^{-1} \dot{\phi} \dot{\phi}$, where $g$ denotes the covariant metric $g_{i j}$. As the $\phi_{i j}$ 's enter only through their time derivatives, their conjugate momenta $\Pi^{i j}$ are constants of the motion in this $|\ell| \leq 1$ truncation. Eliminating the $\phi$ 's in terms of the 
constant momenta $\Pi$ yields

$$
V_{\phi}(g) \propto+g_{i j} g_{k l} \Pi^{i k} \Pi^{j l}
$$

This potential can be identified with the leading (weight-2) gravitational potential, if we identify the structure constants $C^{i}{ }_{j k}$ defined by $d \theta^{i}=$ $C^{i}{ }_{j k} \theta^{j} \wedge \theta^{k}$, with the momenta conjugate to $\phi_{i j}$ as

$$
\Pi^{i j}=\varepsilon^{k l(i} C^{j)} k l
$$

Consequently, the BKL dynamics at each spatial point is equivalent to the $|\ell| \leq 1$ truncation of the $A E_{3}$-invariant dynamics defined by (6.77). The fields $\phi_{i j}(t)$ parametrizing the components of the $A E_{3}$ coset element along the $\ell=1$ generators are canonically conjugate to the structure constants $C^{i}{ }_{j k}$. The proper physical interpretation of the higher level fields remains yet to be found.

Varying (6.77) w.r.t. the lapse function $n$ gives rise to the constraint that the coset Lagrangian vanish. Defining the canonical momenta

$$
\pi_{a}:=\frac{\delta \mathcal{L}}{\delta \dot{\beta}^{a}}=n^{-1} G_{a b} \dot{\beta}^{b}
$$

and the (non-canonical) momentum-like variables

$$
\Pi_{\alpha, s}:=\frac{\delta \mathcal{L}}{\delta j_{\alpha, s}}=\frac{1}{2} n^{-1} \exp (2 \alpha(\beta)) j_{\alpha, s}
$$

and recalling the equivalence of the Cartan Killing and superspace metrics noted above, we are led to the Hamiltonian constraint of the $\sigma$-model, which is given by

$$
\mathcal{H}\left(\beta^{a}, \pi_{a}, \ldots\right)=\frac{1}{2} G^{a b} \pi_{a} \pi_{b}+\sum_{\alpha \in \Delta_{+}} \sum_{s=1}^{\operatorname{mult}(\alpha)} \exp (-2 \alpha(\beta)) \Pi_{\alpha, s} \Pi_{\alpha, s}
$$

where $\beta^{a}, \pi_{a}$ are now the diagonal CSA degrees of freedom, and the dots stand for infinitely many off-diagonal (Iwasawa-type) canonical variables, on which the $\Pi_{\alpha, s}$ depend.

The evident similarity of (3.38) and (6.84) is quite striking, but at this point we can only assert that the two expressions coincide asymptotically, when they both reduce to a relativistic billiard. Namely, because the coefficients of the exponentials in (6.84) are non-negative, we can apply exactly the same reasoning as for the gravitational billiards in section 3.1. One then finds that the off-diagonal components $\nu_{\alpha, s}$ and the momentum-like variables $\Pi_{\alpha, s}$ get frozen asymptotically (again, we may invoke the imagery of a mountainscape, now defined by exponential potentials for all roots). 
In the present KM setup, all the walls enter on the same footing; there is nothing left of the distinctions between different types of walls (symmetry, gravitational, electric, and so on). The only important characteristic of a wall is its height ht $\alpha \equiv n_{1}+n_{2}+\cdots$ for a root decomposed along simple roots as $\alpha=n_{1} \alpha_{1}+n_{2} \alpha_{2}+\cdots$. The asymptotic Hamiltonian hence is dominated by the walls associated to the simple roots:

$$
\mathcal{H}_{\infty}(\beta, \pi)=\frac{1}{2} \pi^{a} \pi_{a}+\sum_{i=1}^{r} \Theta_{\infty}\left(-2 \alpha_{i}(\beta)\right)
$$

where the sum is over the simple roots only, and the motion of the $\beta^{a}$ is confined to the fundamental Weyl chamber $\alpha_{i}(\beta) \geq 0$.

The billiard picture for pure gravity in four dimensions is now readily understood in terms of the Weyl group of $A E_{3},{ }^{64}$ which is just the modular group $P G L(2, \mathbb{Z}),{ }^{18}$ and the simple roots of $A E_{3}$. For the $\mathfrak{s l}(3, \mathbb{R})$ subalgebra, which has two simple roots, the Weyl group is the permutation group on three objects. The two hyperplanes orthogonal to these simple roots can be identified with the symmetry (centrifugal) walls. The third simple root extending (5.56) to the full rank 3 algebra (5.55) can be identified the dominant curvature (gravitational) wall.

To conclude: in the limit where one goes to infinity in the Cartan directions, the dynamics of the Cartan degrees of freedom of the coset model become equivalent to a billiard motion within the Weyl chamber, subject to the zero-energy constraint $\mathcal{H}_{\infty}(\beta, \pi)=0$. Therefore, in those cases where the gravitational billiards of section 3.1 are of KM-type, they are asymptotically equivalent to the $\mathrm{KM} \sigma$-models over $G / K(G)$.

\section{Finale: $E_{10}$ - the ultimate symmetry?}

There can be little doubt that the algebra, which from many points is the most intriguing and most beautiful, is the maximal rank hyperbolic KM algebra $E_{10}$, which is an infinite dimensional extension of the better known finite dimensional exceptional Lie algebras $E_{6}, E_{7}$ and $E_{8} .{ }^{72}$ There are two other rank-10 hyperbolic $\mathrm{KM}$ algebras $D E_{10}$ and $B E_{10}$ (respectively related to type I supergravity, and Einstein Maxwell supergravity in ten dimensions), but they appear to be less distinguished. The emergence of $E_{10}$ in the reduction of $D=11$ supergravity to one dimension had first been conjectured in. ${ }^{70} \mathrm{~A}$ crucial new feature of the scheme proposed here, which is based on a hyperbolic $\sigma$-model defined by means of the geodesic action (6.77) is that it retains a residual spatial dependence, which on the 
$\sigma$-model side is supposed 'to be spread' over the whole $E_{10}$ Lie algebra. Thereby all degrees of freedom of the original theory should still be there, unlike for a bona fide reduction to one dimension.

Just like $A E_{3}$ the KM algebra $E_{10}$ algebra is recursively defined via its Chevalley-Serre presentation in terms of generators and relations and its Dynkin diagram which we give below.

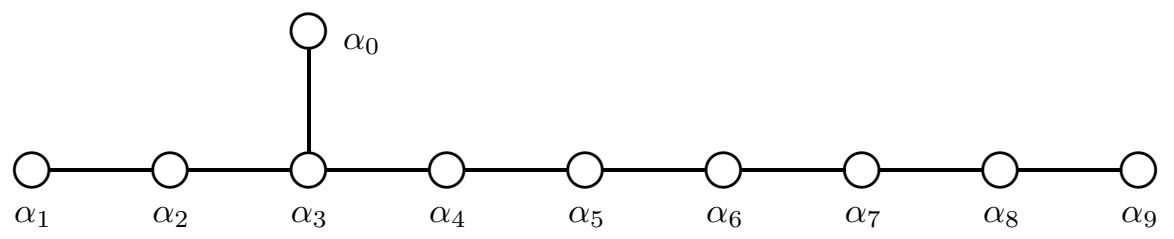

The nine simple roots $\alpha_{1}, \ldots, \alpha_{9}$ along the horizontal line generate an $A_{9} \equiv$ $\mathfrak{s l}(10, \mathbb{R})$ subalgebra. One of the reasons why $E_{10}$ is distinguished is that its root lattice is the unique even self-dual Lorentzian lattice $\mathrm{II}_{1,9}$ (such lattices exist only in dimensions $d=2+8 n .{ }^{73}$ )

For the corresponding $\sigma$-model a precise identification can be made between the purely $t$-dependent $\sigma$-model quantities obtained from the geodesic action on the $E_{10} / K\left(E_{10}\right)$ coset space on the one hand, and certain fields of $D=11$ supergravity and their spatial gradients evaluated at a given, but arbitrarily chosen spatial point on the other. ${ }^{25,27}$ The simple and essentially unique geodesic Lagrangian describing a null world line in the infinite-dimensional coset manifold $E_{10} / K\left(E_{10}\right)$ thus reproduces the dynamics of the bosonic sector of eleven-dimensional supergravity in the vicinity of a space-like singularity. This result can be extended to massive IIA supergravity, ${ }^{26}$ where also parts of the fermionic sector were treated for the first time, and to IIB supergravity in. ${ }^{28}$ Related results had been previously obtained in the framework of $E_{11} \cdot{ }^{74,75,76}$

A main ingredient in the derivation of these results is the level decomposition of $E_{10}$ w.r.t. the $A_{9}, D_{9}$, and $A_{8} \times A_{1}$ subalgebras of $E_{10}$, respectively, which generalizes the $\mathfrak{s l}(3, \mathbb{R})$ decomposition of $A E_{3}$ made in section 5 . In all cases, one obtains precisely the field representation content of the corresponding supergravity theories at the lowest levels, and for all these decompositions, the bosonic supergravity equations of motion, when restricted to zeroth and first order spatial gradients, match with the corresponding $\sigma$-model equations of motion at the lowest levels. In particular, the self-duality of the five-form field strength in type IIB supergravity is 
implied by the dynamical matching between the $E_{10} / K\left(E_{10}\right) \sigma$-model and the supergravity equations of motion, and does not require local supersymmetry or some other extraneous argument for its explanation.

Combining the known results, we can summarize the correspondence between the maximally supersymmetric theories and the maximal rank regular subalgebras of $E_{10}$ as follows

$$
\begin{aligned}
A_{9} \subset E_{10} & \Longleftrightarrow \quad D=11 \text { supergravity } \\
D_{9} \subset E_{10} & \Longleftrightarrow \quad \text { massive IIA supergravity } \\
A_{8} \times A_{1} \subset E_{10} & \Longleftrightarrow \quad \text { IIB supergravity }
\end{aligned}
$$

The decompositions of $E_{10}$ w.r.t. its other rank 9 regular subalgebras $A_{D-2} \times E_{11-D}$ (for $D=3, \ldots, 9$ ) will similarly reproduce the representation content of maximal supergravities in $D$ space-time dimensions as the lowest level representations.

We conclude by repeating the main challenge that remains: one must extend these correspondences to higher levels and spatial gradients, and find a physical interpretation for the higher level representations, whose number exhibits an exponential growth similar to the growth in the number of excited string states (see, however, ${ }^{29}$ for recent progress concerning the link between higher order $\mathrm{M}$ Theory corrections and the $E_{10}$ root lattice). Because this will inevitably require (or entail) a detailed understanding of indefinite and hyperbolic KM algebras, it might also help in solving the core problem of the theory of Kac Moody algebras, a problem that has vexed almost a generation of researchers.

\section{Acknowledgments}

This work was supported in part by the European Network HPRN-CT2000-00122 and by the the German Israeli Foundation (GIF) Project Nr. I. 645 130-14-1999. It is a great pleasure to thank T. Damour, T. Fischbacher, M. Henneaux and A. Kleinschmidt for enjoyable collaborations and innumerable discussions that have shaped my understanding of the results reported here, and I.H.E.S., Bures-sur-Yvette, for continued support during several visits there. I am also very grateful to F. Englert, A. Feingold, A. Giveon, L. Houart and E. Rabinovici for enlightening discussions at various earlier stages of this work. 


\section{References}

1. H.A. Buchdahl, "Reciprocal static solutions of the equation $G_{\mu \gamma}=0$ ", Quart. J. Math. Oxford 5, 116 (1954)

2. J. Ehlers, Dissertation Hamburg University (1957)

3. R. Geroch, "A method for generating solutions of Einstein's equations", J. Math. Phys. 12, 918 (1971);

"A method for generating solutions of Einstein's equations. 2", J. Math. Phys. 13, 394 (1972).

4. W. Dietz and C. Hoenselars (eds.), Solutions of Einstein's equations: techniques and results (Springer, 1984)

5. D. Kramer et al.: "Exact Solutions of Einstein's Field Equations", Cambridge University Press

6. E. Cremmer and B. Julia, "The SO(8) supergravity", Nucl. Phys. B159 (1979) 141

7. B. Julia, LPTENS 80/16, Invited paper presented at Nuffield Gravity Workshop, Cambridge, Eng., Jun 22 - Jul 12, 1980.

8. B. Julia, in the Proceedings of the Johns Hopkins Workshop on Current Problems in Particle Physics "Unified Theories and Beyond" (Johns Hopkins University, Baltimore, 1984).

9. P. Breitenlohner and D. Maison, "On the Geroch group", Ann. Inst. Henri Poincaré 46, 215 (1986).

10. P. Breitenlohner, D. Maison and G. W. Gibbons, "Four-Dimensional Black Holes From Kaluza-Klein Theories," Commun. Math. Phys. 120, 295 (1988).

11. H. Nicolai, in "Recent Aspects of Quantum Fields", Proceedings Schladming 1991, Lecture Notes in Physics (Springer Verlag, 1991)

12. W. Kinnersley and D. Chitre, J. Math. Phys. 18 (1977) 1583; 19 (1978) 1926 and 2037

13. D. Maison, Phys. Rev. Lett. 41 (1978) 521

14. V. Belinskii and V. Zakharov, "Integration of Einstein's equations by means of inverse scattering problem technique and construction of exact soliton solutions, Sov. Phys. JETP 48 (1978) 985

15. V.G. Kac, Infinite Dimensional Lie Algebras, 3rd edn., Cambridge University Press, 1990

16. R.V. Moody and A. Pianzola, Lie Algebras with Triangular Decomposition, Wiley, New York, 1995

17. P. Goddard and D.I. Olive, "Kac-Moody and Virasoro algebras in relation to quantum physics", Int. J. Mod . Phys. A1, 303 (1986)

18. A.J. Feingold and I.B. Frenkel, "A hyperbolic Kac-Moody algebra and the theory of Siegel modular form of genus 2", Math. Ann. 263, 87 (1983)

19. E. Cremmer, B. Julia and J. Scherk, "Supergravity Theory In 11 Dimensions," Phys. Lett. B 76, 409 (1978).

20. K. Kuchar, "Canonical quantum gravity", gr-qc/9304012

21. A. Giveon, M. Porrati and E. Rabinovici, "Target space duality in string theory", Phys. Rep. 244 (1994) 77, hep-th/9401139

22. C.M. Hull and P.K. Townsend, "Unity of superstring dualities", Nucl. Phys. 
B438 (1995) 109, hep-th/9410167

23. N.A. Obers and B. Pioline, "U Duality and M Theory", Phys. Rep. 318 (1999) 113, hep-th/9809039

24. T. Damour and M. Henneaux, "E(10), BE(10) and arithmetical chaos in superstring cosmology," Phys. Rev. Lett. 86, 4749 (2001) [arXiv:hepth/0012172].

25. T. Damour, M. Henneaux and H. Nicolai, $E_{10}$ and a small tension expansion of M Theory, Phys. Rev. Lett. 89 (2002): 221601, hep-th/0207267

26. A. Kleinschmidt and H. Nicolai, JHEP 0407:041 (2004) hep-th/0407101

27. T. Damour and H. Nicolai, "Eleven dimensional supergravity and the $E_{10} / K\left(E_{10}\right)$ sigma-model at low $A_{9}$ levels", hep-th/0410245

28. A. Kleinschmidt and H. Nicolai, "IIB Supergravity and $E_{10}$ ", Phys. Lett. B606 (2005) 391, hep-th/0411225

29. T. Damour and H. Nicolai, Higher order M theory corrections and the Kac Moody algebra $E_{10}$, hep-th/0504153

30. J. Brown, O. Ganor and C. Helfgott, $M$ theory and $E_{10}$ : Billiards, Branes, and Imaginary Roots, JHEP 0408:063 (2004)hep-th/0401053;

31. J. Brown, S. Ganguli, O. Ganor and C. Helfgott, E(10) orbifolds, hep-th/0409037

32. P. C. West, " $E_{11}$ and M Theory", Class. Quant. Grav. 18, 4443-4460 (2001), [arXiv:hep-th/0104081]

33. P.C. West, " $E_{11}$ and central charges", Phys. Lett. B575 (2003) 333, hep-th/0307098; "Brane dynamics, central charges and $E(11)$, hep-th/0412336

34. P.C. West, "Some simple predictions for $E(11)$ symmetry", hep-th/0407088

35. A. Kleinschmidt and P.C. West, "Representations of $G^{+++}$and the role of spacetime", JHEP 0402 (2004) 033, hep-th/0312247

36. M. Gaberdiel, D.I. Olive and P.C. West, "A class of Lorentzian Kac Moody algebras", Nucl. Phys. B645 (2002) 403, hep-th/0205068

37. F. Englert, L. Houart, A. Taormina and P.C. West, "The symmetry of M theories", JHEP 0309 (2003) 020, hep-th/0304206

38. F. Englert and L. Houart, $G^{+++}$invariant formulation of gravity and M-theories: Exact BPS solutions, JHEP 0401 (2004) 002 [arXiv:hepth/0311255]; $G^{+++}$invariant formulation of gravity and M-theories: Exact intersecting brane solutions, JHEP 0405 (2004) 059 [arXiv:hep-th/0405082].

39. T. Curtright, Phys. Lett. B165 (1985) 304

40. N.A. Obers, B. Pioline and E. Rabinovici, "M-theory and U-duality on $T^{d}$ with gauge backgrounds, Nucl. Phys. B 525 (1998) 163, hep-th/9712084

41. C.M. Hull, "Strongly coupled gravity and duality", Nucl. Phys. B 583 (2000) 237, hep-th/0004195

42. X. Bekaert, N. Boulanger and M. Henneaux, "Consistent deformations of dual formulations of linearized gravity: a no go result", Phys. Rev. D67 (2003) 044010, hep-th/0210278

43. M. Henneaux and C. Teitelboim, "Duality in linearized gravity", Phys. Rev. D71: 024018 (2005), gr-qc/0408101

44. G. Neugebauer and D. Kramer, Annalen der Physik 24 (1969) 62 
45. H. Nicolai and A. Nagar, "Infinite dimensional symmetries in gravity", in Gravitational Waves, eds. I. Ciufolini, V. Gorini, U. Moschella and F. Fré, IoP, Bristol, 2001

46. H. Nicolai, "A Hyperbolic Lie Algebra From Supergravity," Phys. Lett. B276, 333 (1992).

47. V.A. Belinskii, I.M. Khalatnikov and E.M. Lifshitz, "Oscillatory approach to a singular point in the relativistic cosmology," Adv. Phys. 19, 525 (1970).

48. V.A. Belinskii, I.M. Khalatnikov and E.M. Lifshitz, "Construction of a general cosmological solution of the Einstein equation with a time singularity", Sov. Phys. JETP 35, 838-841 (1972).

49. V.A. Belinskii, I.M. Khalatnikov and E.M. Lifshitz, "A general solution of the Einstein equations with a time singularity," Adv. Phys. 31, 639 (1982).

50. C.W. Misner, "Mixmaster universe," Phys. Rev. Lett. 22, 1071-1074 (1969).

51. C. W. Misner, "Quantum Cosmology. 1," Phys. Rev. 186, 1319 (1969); "Minisuperspace," In *J R Klauder, Magic Without Magic*, San Francisco 1972, 441-473.

52. D.M. Chitre, Ph. D. thesis, University of Maryland, 1972.

53. M. P. Ryan, "Hamiltonian cosmology," Springer-Verlag, Heidelberg (1972).

54. M. P. Ryan and L. C. Shepley, "Homogeneous Relativistic Cosmologies," Princeton, USA: Univ. Pr. (1975) 320 P. (Princeton Series In Physics).

55. R. T. Jantzen, "Spatially homogeneous dynamics: A unified picture," arXiv:gr-qc/0102035.

56. B. K. Berger, "Numerical Approaches to Spacetime Singularities," arXiv:grqc/0201056.

57. B. K. Berger and V. Moncrief, "Signature for local mixmaster dynamics in $U(1)$ symmetric cosmologies", Phys. Rev. D62: 123501 (2000) arXiv:grqc/0006071

58. N. J. Cornish and J. J. Levin, "The mixmaster universe is chaotic," Phys. Rev. Lett. 78, 998 (1997) [arXiv:gr-qc/9605029]; "The mixmaster universe: A chaotic Farey tale," Phys. Rev. D 55, 7489 (1997) [arXiv:gr-qc/9612066].

59. B. K. Berger, D. Garfinkle, J. Isenberg, V. Moncrief and M. Weaver, "The singularity in generic gravitational collapse is spacelike, local, and oscillatory," Mod. Phys. Lett. A 13, 1565 (1998) [arXiv:gr-qc/9805063].

60. L. Andersson and A.D. Rendall, "Quiescent cosmological singularities," Commun. Math. Phys. 218, 479-511 (2001) [arXiv:gr-qc/0001047].

61. E. Anderson, "Strong-coupled relativity without relativity," arXiv:grqc/0205118.

62. T. Damour and M. Henneaux, "Chaos in superstring cosmology," Phys. Rev. Lett. 85, 920 (2000) [arXiv:hep-th/0003139]; [See also short version in Gen. Rel. Grav. 32, 2339 (2000).]

63. T. Damour and M. Henneaux, "Oscillatory behaviour in homogeneous string cosmology models," Phys. Lett. B 488, 108 (2000) [arXiv:hep-th/0006171].

64. T. Damour, M. Henneaux, B. Julia and H. Nicolai, "Hyperbolic Kac-Moody algebras and chaos in Kaluza-Klein models," Phys. Lett. B 509, 323 (2001) [arXiv:hep-th/0103094].

65. T. Damour, M. Henneaux and H. Nicolai, Class. Quant. Grav. 20 (2003) 
R145-R200, hep-th/0212256

66. V.D. Ivashchuk and V.N. Melnikov, "Billiard Representation For Multidimensional Cosmology With Multicomponent Perfect Fluid Near The Singularity," Class. Quantum Grav. 12, 809 (1995).

67. V. D. Ivashchuk and V. N. Melnikov, "Billiard representation for multidimensional cosmology with intersecting p-branes near the singularity," J. Math. Phys. 41, 6341 (2000) [arXiv:hep-th/9904077].

68. T. Damour, M. Henneaux, A. D. Rendall and M. Weaver, "Kasner-like behaviour for subcritical Einstein-matter systems," arXiv:gr-qc/0202069, to appear in Ann. Inst. H. Poincaré.

69. T. Damour, S. de Buyl, M. Henneaux and C. Schomblond, "Einstein billiards and overextensions of finite-dimensional simple Lie algebras," arXiv:hepth/0206125.

70. B. Julia, in Lectures in Applied Mathematics, AMS-SIAM, vol 21 (1985), p.355.

71. H. Nicolai and T. Fischbacher, in: Kac Moody Lie Algebras and Related Topics, eds. N. Sthanumoorthy and K.C. Misra, Contemporary Mathematics 343, American Mathematical Society, 2004, hep-th/0301017

72. J. E. Humphreys, "Introduction to Lie Algebras and Representation Theory", Graduate Texts in Mathematics 9, Springer Verlag, 1980

73. J.H. Conway and N.J.A. Sloane, "Sphere Packings, Lattices and Groups", Grundlehren der mathematischen Wissenschaften 290, 2nd edition, Springer Verlag, 1991

74. I. Schnakenburg and P. C. West, "Kac-Moody symmetries of IIB supergravity," Phys. Lett. B 517, 421 (2001) [arXiv:hep-th/0107181].

75. I. Schnakenburg and P. C. West, "Massive IIA supergravity as a non-linear realisation," Phys. Lett. B 540, 137 (2002) [arXiv:hep-th/0204207].

76. A. Kleinschmidt, I. Schnakenburg and P. West, Very-extended Kac-Moody algebras and their interpretation at low levels, Class. Quant. Grav. 21 (2004) 2493 [arXiv:hep-th/0309198]. 